\title{
Inibição micelial in vitro de Sclerotinia sclerotiorum por fungicidas
}

\author{
Ciro Hideki Sumida ${ }^{1}$, Marcelo Giovanetti Canteri ${ }^{1}$, Douglas Casaroto Peitl ${ }^{1}$, \\ Idenize Pedrina Orsini ${ }^{1}$, Fabiana Tibolla ${ }^{1}$, Felipe André Araújo ${ }^{1}$, Debora Fonseca Chagas ${ }^{1}$
}

\author{
${ }^{1}$ Departamento de Agronomia - Universidade Estadual de Londrina (UEL), Rod. Celso Garcia Cid. (PR 445), Km 380, CP 6001, CEP 86051-990. \\ Londrina, PR. \\ Autor para correspondência: Ciro Hideki Sumida (cirosumida@hotmail.com) \\ Data de chegada: 23/08/2013. Aceito para publicação em: 18/02/2014.
}

Sclerotinia sclerotiorum (Lib.) de Bary, considerado um fitopatógeno altamente destrutivo, tem causado perdas significativas na produção agrícola no mundo todo. O controle do fungo depende da associação de práticas culturais como controle biológico (GÖRGEN et al., 2009), controle genético, embora este ainda em estudo (BARDIN e HUANG, 2001) e controle químico (COSTA e COSTA, 2004).

A aplicação de fungicidas pode auxiliar no controle da doença, reduzindo, significativamente, o número de escleródios viáveis. Esta estratégia pode ser usada como medida preventiva por meio de tratamento de sementes e aplicações durante o período vegetativo ou reprodutivo (MUELLER et al., 2002). Por outro lado, existem relatos sobre o uso de fungicidas no controle de $S$. sclerotiorum com resultados inconsistentes (FERRAZ et al., 2003) devido a dificuldades em conseguir uma boa cobertura com a aplicação de fungicidas e coincidir a época de aplicação com a liberação dos ascósporos (MORTON e HALL, 1989). Avaliações da eficácia in vitro de fungicidas em diferentes tipos de inóculo de $S$. sclerotiorum como micélio e escleródios são importantes para relacionar os resultados nas diferentes fases do seu ciclo de vida.

No presente trabalho, avaliou-se in vitro a eficiência de vinte sete fungicidas, para inibir o crescimento micelial, produção e viabilidade dos escleródios de S. sclerotiorum. A maioria dos fungicidas foi testadas visando a cultura da soja, nas concentrações de 0,$1 ; 1,0 ; 10 ; 100$ e 1000 $\mu \mathrm{L}$ de produto formulado/1L de meio de cultura BDA (batata-dextroseágar). O isolado de S. sclerotiorum utilizado no teste provém de campos de produção de soja do município de Mauá da Serra-PR. O bioensaio foi realizado por meio da técnica de homogeneização de fungicida em meio de cultura BDA (batata-dextrose-ágar) e vertidos em placas de Petri, no qual foram transferidos discos de colônia de S. sclerotiorum no centro de cada placa. Após $72 \mathrm{~h}$ de incubação em câmaras BOD à temperatura de $20^{\circ} \mathrm{C} \pm 2$, mensurou-se o diâmetro médio das colônias, comparando-as ao crescimento em placas sem tratamento fungicida. Posteriormente, após 21 dias de incubação, realizou-se a contagem do número total de escleródios, a pesagem e o teste de viabilidade em meio de cultura Neon (PERES et al., 2002). Os dados obtidos foram submetidos à análise de variância e as médias comparadas pelo teste de Scott-Knott, em nível de 5\% de probabilidade de erro, utilizando o programa SASM-Agri (CANTERI et al., 2001). Realizaram-se análises estatísticas separadamente para cada concentração de fungicida.

Entre os produtos avaliados, o fluazinam foi o mais eficiente para inibição do crescimento micelial de S. sclerotiorum em todas as concentrações, de 0,1 a $1000 \mu \mathrm{L} / \mathrm{L}$ (Tabela 1). Na concentração de $1000 \mu \mathrm{L} / \mathrm{L}$, todos os fungicidas apresentaram $100 \%$ de inibição (dados não apresentados), com exceção do fungicida pencicurom. Na maioria dos tratamentos, à medida que a concentração dos fungicidas foi reduzida, também observou-se a redução da porcentagem de inibição micelial. A produção de escleródios variou em função da concentração do produto, porém a viabilidade dos mesmos não foi afetada pela maioria dos fungicidas, independente do produto ou concentração, ou seja, os escleródios que cresceram sobre o meio de cultura tratados com os diferentes fungicidas apresentaram viabilidade de $100 \%$. Resultados diferentes podem ser observados quando os fungicidas são aplicados sobre os escleródios, como relatados por COSTA e COSTA (2004) onde os tratamentos com aplicação de fluazinam, procimidone e iprodione apresentaram 100, 95 e 85\% de inibição da germinação carpogênica.

A inibição do desenvolvimento dos escleródios ou sua inviabilização são os fatores mais importantes para reduzir os danos causados pelo patógeno, pois segundo FERRAZ et al. (2003), em diversas culturas tem sido difícil o controle da doença devido a sua capacidade em formar os escleródios, que garantem sua sobrevivência por vários anos. Em condições de campo, a utilização do fungicida com penetração no dossel da planta atingindo a superfície do solo é determinante na redução da germinação dos escleródios, consequentemente, diminuindo a produção de apotécios, causando atraso no desenvolvimento do mesmo e reduzindo a incidência da doença (MUELLER et al., 2002).

OLIVEIRA et al. (1999) desenvolveram um teste semelhante de inibição micelial de $S$. sclerotiorum com fluazinam, procimidona, carbendazim, iprodiona e outros, nas concentrações de 1;10 e $100 \mu \mathrm{L} / \mathrm{L}$. Observaram inibição de $100 \%$ com todos fungicidas e concentrações. Em relação a produção de escleródios somente o fluazinam inibiu a produção nas três concentrações, corroborando com os dados do presente trabalho. Trabalho similar foi desenvolvido por PAULA JUNIOR et al. (2009) com os fungicidas fluazinam e procimidona nas mesmas concentrações de 0,1 a $1000 \mu \mathrm{L} / \mathrm{L}$ utilizadas nesse trabalho, onde relataram maior inibição micelial pelo fungicida fluazinam nas concentração de 0,1 até $100 \mu \mathrm{L} / \mathrm{L}$ e somente a $1000 \mu \mathrm{L} / \mathrm{L}$ foi observado resultado semelhante para os dois fungicidas ( $100 \%$ de inibição).

Os testes realizados mostraram resultados de eficiência dos fungicidas em relação à $S$. sclerotiorum em condições controladas, mas é importante ressaltar a diferença das avaliações in vitro em relação às de campo. Em condições de campo, o fluazinam e procimidona são relatados como eficientes fungicidas para o controle do patógeno, mas vários outros fungicidas como os testados no presente trabalho não são avaliados, principalmente em relação à avaliação do retorno econômico que podem apresentar em comparação com o fluazinam e procimidona. 
Tabela 1. Porcentagem de inibição do crescimento micelial de Sclerotina sclerotiorum tratados com fungicidas por meio de homogeneização no meio de cultura fundente BDA (batata-dextrose-ágar) em placas de Petri. Londrina - PR, 2010.

\begin{tabular}{|c|c|c|c|c|c|c|c|c|c|}
\hline $\begin{array}{c}\text { Tratamentos } \\
\text { (ingrediente ativo) }\end{array}$ & Concentração & \multicolumn{2}{|c|}{$0,1 \mu \mathrm{L} / \mathrm{L}$} & \multicolumn{2}{|c|}{$1,0 \mu \mathrm{L} / \mathrm{L}$} & \multicolumn{2}{|c|}{$10 \mu \mathrm{L} / \mathrm{L}$} & \multicolumn{2}{|l|}{$100 \mu \mathrm{L} / \mathrm{L}$} \\
\hline testemunha & 0 & - & & - & & - & & - & \\
\hline azoxistrobina - ciproconazol & $200-80 \mathrm{~g} / \mathrm{L}$ & 2,9 & $\mathrm{~g}$ & 20,0 & $\mathrm{i}$ & 30,8 & $\mathrm{~g}$ & 84,9 & $\mathrm{~d}$ \\
\hline difenoconazol & $250 \mathrm{~g} / \mathrm{L}$ & 13,4 & $\mathrm{f}$ & 54,5 & $\mathrm{f}$ & 94,1 & $\mathrm{~b}$ & 100,0 & $\mathrm{a}$ \\
\hline flutriafol & $125 \mathrm{~g} / \mathrm{L}$ & 2,4 & $\mathrm{~g}$ & 0,0 & $\mathrm{k}$ & 5,2 & $\mathrm{i}$ & 16,2 & $\mathrm{e}$ \\
\hline ciproconazol & $100 \mathrm{~g} / \mathrm{L}$ & 15,7 & $\mathrm{f}$ & 33,1 & $\mathrm{~h}$ & 43,1 & $\mathrm{f}$ & 100,0 & $\mathrm{a}$ \\
\hline metalaxil-m & $10-25 \mathrm{~g} / \mathrm{L}$ & 21,2 & $\mathrm{f}$ & 100,0 & a & 100,0 & a & 100,0 & $\mathrm{a}$ \\
\hline tebuconazole & $200 \mathrm{~g} / \mathrm{L}$ & 0,9 & $\mathrm{~g}$ & 40,2 & $\mathrm{~g}$ & 94,6 & $\mathrm{~b}$ & 100,0 & a \\
\hline triadimenol & $150 \mathrm{~g} / \mathrm{L}$ & 0,7 & $\mathrm{~g}$ & 11,0 & $\mathrm{j}$ & 25,6 & $\mathrm{~h}$ & 82,4 & $\mathrm{~d}$ \\
\hline pencicurom & $250 \mathrm{~g} / \mathrm{L}$ & 0,0 & $\mathrm{~g}$ & 0,0 & $\mathrm{k}$ & 0,0 & $\mathrm{j}$ & 0,0 & f \\
\hline miclobutanil & $250 \mathrm{~g} / \mathrm{L}$ & 0,0 & $\mathrm{~g}$ & 11,1 & $\mathrm{j}$ & 43,2 & $\mathrm{f}$ & 95,2 & $\mathrm{~b}$ \\
\hline piraclostrobina - epoxiconazol & $133-50 \mathrm{~g} / \mathrm{L}$ & 15,5 & $\mathrm{f}$ & 33,1 & $\mathrm{~h}$ & 76,6 & d & 100,0 & $\mathrm{a}$ \\
\hline cloreto de benzalcônio & $100 \mathrm{~g} / \mathrm{L}$ & 0,0 & $\mathrm{~g}$ & 0,0 & $\mathrm{k}$ & 0,0 & $\mathrm{j}$ & 17,6 & $\mathrm{e}$ \\
\hline picoxystrobina - ciproconazol & $200-80 \mathrm{~g} / \mathrm{L}$ & 27,4 & $\mathrm{e}$ & 66,1 & d & 100,0 & a & 100,0 & $\mathrm{a}$ \\
\hline hidróxido de fentina & $400 \mathrm{~g} / \mathrm{L}$ & 0,0 & $\mathrm{~g}$ & 65,0 & d & 100,0 & a & 100,0 & $\mathrm{a}$ \\
\hline trifloxistrobina - tebuconazole & $100-200 \mathrm{~g} / \mathrm{L}$ & 11,0 & $\mathrm{~g}$ & 32,9 & $\mathrm{~h}$ & 83,1 & $\mathrm{c}$ & 100,0 & $\mathrm{a}$ \\
\hline procimidona & $500 \mathrm{~g} / \mathrm{kg}$ & 19,9 & $\mathrm{f}$ & 81,7 & $\mathrm{c}$ & 100,0 & a & 100,0 & $\mathrm{a}$ \\
\hline tolilfluanida & $500 \mathrm{~g} / \mathrm{kg}$ & 48,9 & $\mathrm{c}$ & 100,0 & a & 100,0 & a & 100,0 & $\mathrm{a}$ \\
\hline tiofanato-metílico & $700 \mathrm{~g} / \mathrm{kg}$ & 15,7 & $\mathrm{f}$ & 42,8 & $\mathrm{~g}$ & 100,0 & a & 100,0 & $\mathrm{a}$ \\
\hline clorotalonil & $750 \mathrm{~g} / \mathrm{kg}$ & 37,1 & d & 83,4 & $\mathrm{c}$ & 100,0 & a & 100,0 & $\mathrm{a}$ \\
\hline fluazinam & $500 \mathrm{~g} / \mathrm{L}$ & 100,0 & a & 100,0 & $\mathrm{a}$ & 100,0 & a & 100,0 & $\mathrm{a}$ \\
\hline iprodiona & $500 \mathrm{~g} / \mathrm{L}$ & 20,4 & $\mathrm{f}$ & 52,7 & $\mathrm{f}$ & 100,0 & $\mathrm{a}$ & 100,0 & $\mathrm{a}$ \\
\hline azoxistrobina & $500 \mathrm{~g} / \mathrm{kg}$ & 47,9 & $\mathrm{c}$ & 59,4 & $\mathrm{e}$ & 100,0 & a & 100,0 & $\mathrm{a}$ \\
\hline tiabendazol & $485 \mathrm{~g} / \mathrm{L}$ & 21,9 & $\mathrm{f}$ & 36,8 & $\mathrm{~h}$ & 100,0 & a & 100,0 & $\mathrm{a}$ \\
\hline carbendazim & $500 \mathrm{~g} / \mathrm{L}$ & 37,2 & d & 100,0 & a & 100,0 & a & 100,0 & $\mathrm{a}$ \\
\hline carbendazim - tiram & $150-350 \mathrm{~g} / \mathrm{L}$ & 15,1 & $\mathrm{f}$ & 90,0 & $\mathrm{~b}$ & 100,0 & a & 100,0 & $\mathrm{a}$ \\
\hline metconazol & $90 \mathrm{~g} / \mathrm{L}$ & 91,1 & $\mathrm{~b}$ & 100,0 & a & 100,0 & a & 100,0 & $\mathrm{a}$ \\
\hline piraclostrobina & $250 \mathrm{~g} / \mathrm{L}$ & 35,5 & d & 49,5 & $\mathrm{f}$ & 83,6 & $\mathrm{c}$ & 88,9 & $\mathrm{c}$ \\
\hline tiametoxam - ciproconazol & $300-300 \mathrm{~g} / \mathrm{kg}$ & 14,1 & $\mathrm{f}$ & 33,2 & $\mathrm{~h}$ & 100,0 & a & 100,0 & $\mathrm{a}$ \\
\hline CV (\%) & & 29,6 & & 9,1 & & 3,2 & & 2,5 & \\
\hline
\end{tabular}

Médias seguidas pela mesma letra na coluna não diferem entre si pelo teste Scott-knott a nível de $5 \%$ de significância. $(\mu \mathrm{L} / \mathrm{L}$ ) microlitros de produto formulado por litro de meio de cultura. 